\title{
DIABETES Weight status affects mortality risk
}

People whose BMI is in the normal range at the time that they are identified as having diabetes mellitus have double the risk of mortality over an 18 year follow-up period compared with people who have a BMI in the overweight or obese range when diabetes mellitus is identified, according to researchers in the USA.

"In our previous research the predominant risk factor for diabetes mellitus was overweight and obesity, but we noted that some people developed diabetes mellitus without this very strong risk factor," explains lead researcher Mercedes Carnethon of Northwestern University, Chicago, USA. "We wanted to learn more about the health outcomes of this unique subset of people with diabetes mellitus."

The researchers carried out a pooled analysis of five longitudinal cohort studies that included 2,625 participants. The cohorts comprised men and women $>40$ years old who developed incident diabetes mellitus, defined as a fasting plasma glucose level of $\geq 7 \mathrm{mmol} / \mathrm{I}$ or by starting to use insulin or oral medication to treat hyperglycaemia.

Participants' BMI was recorded when diabetes mellitus was identified. A BMI of $18.5-24.99 \mathrm{~kg} / \mathrm{m}^{2}$ was considered to represent normal weight and a BMI of $25 \mathrm{~kg} / \mathrm{m}^{2}$ or greater was deemed to represent overweight. Around $12 \%$ of participants were normal weight. "We compared mortality rates of individuals who were normal weight with those who were overweight or obese at the time of diabetes mellitus identification," explains Carnethon.

Patients were followed up from diagnosis of diabetes mellitus until they died, reached the end of surveillance or were lost to follow-up. A total of 449 patients died during 27,125 patient-years of follow-up; 178 deaths were cardiovascular-related and 253 were of noncardiovascular causes. Total mortality in the normal-weight group was 284.8 deaths per 10,000 patient-years, compared with 152.1 deaths per 10,000 patient-years in the overweight or obese group. The normal-weight group had 99.8 cardiovascular-related and 198.1 noncardiovascular deaths per 10,000 years, whereas 67.8 cardiovascular-related and 87.9 noncardiovascular deaths per 10,000 years occurred in the overweight or obese group. For normal-weight individuals, the hazard ratios were $1.52(95 \% \mathrm{Cl}, 0.89-2.58)$ for cardiovascular-related death, $2.32(95 \% \mathrm{Cl}$, 1.55-3.48) for noncardiovascular mortality, and 2.08 (95\% Cl, 1.52-3.48) for total mortality.

The increase in mortality persisted after excluding those who died within the first 2 years of follow-up (who could have had a pre-existing illness) or who lost weight before death. "We had thought that this 'obesity paradox' might occur in patients with diabetes mellitus but were surprised by the strong and consistent finding," observes Carnethon.

The range of data collected in the original studies was limited and the researchers were unable to determine the role of body composition or fat distribution. They were also unable to control for differences in the treatment of diabetes mellitus. "Normal-weight patients might receive less advice on diet and exercise than overweight patients, and less metformin, but could have more sulfonylurea, insulin and hypoglycaemic episodes," comments Jonathan Shaw of Baker IDI Heart and Diabetes Institute, Melbourne, Australia, who was not involved in the study.

The strongest association of normal BMI at diagnosis was with all-cause mortality. Carnethon and colleagues would like to know more about causes of death in normal-weight individuals with diabetes mellitus. "We hope that by leveraging medical record data in future studies we can learn more about this growing subset of patients with diabetes who are not overweight," says Carnethon. More work is needed to understand the mechanisms of disease in this group. "We need to recognise that having diabetes and being normal weight might not be a good starting point," concludes Shaw.

\section{Andy McLarnon}

Original article Carnethon, M. R. et al. Association of weight status with mortality in adults with incident diabetes. JAMA 308, 581-590 (2012) 